\title{
The promoter of $Z m M R P-1$, a maize transfer cell-specific transcriptional activator, is induced at solute exchange surfaces and responds to transport demands
}

\author{
Cristina Barrero • Joaquín Royo • \\ Carmen Grijota-Martinez $\cdot$ Christian Faye $\cdot$ Wyatt Paul $\cdot$ \\ Soledad Sanz • H.-H. Steinbiss • Gregorio Hueros
}

Received: 24 June 2008 / Revised: 15 August 2008 / Accepted: 11 September 2008 / Published online: 2 October 2008

(C) Springer-Verlag 2008

\begin{abstract}
Transfer cells have specializations that facilitate the transport of solutes across plant exchange surfaces. ZmMRP-1 is a maize (Zea mays) endosperm transfer cellspecific transcriptional activator that plays a central role in the regulatory pathways controlling transfer cell differentiation and function. The present work investigates the signals controlling the expression of ZmMRP-1 through the production of transgenic lines of maize, Arabidopsis, tobacco and barley containing ZmMRP-1promoter:GUS reporter constructs. The GUS signal predominantly appeared in regions of active transport between source and sink tissues, including nematode-induced feeding structures and at sites of vascular connection between developing organs and the main plant vasculature. In those cases, promoter induction was associated with the initial developmental stages of transport structures. Significantly, transfer cells also differentiated in these regions suggesting that, independent of
\end{abstract}

C. Barrero · J. Royo · C. Grijota-Martinez · G. Hueros ( $\square)$

Departamento de Biología Celular y Genética,

Universidad de Alcalá, Campus Universitario,

Carretera Madrid-Barcelona Km 33.600,

28871 Alcalá de Henares, Madrid, Spain

e-mail: gregorio.hueros@uah.es

C. Faye $\cdot$ W. Paul

Cereal Functional Analysis Group,

Biogemma SAS, 8 Rue des Frères Lumière,

63028 Clermont-Ferrand Cedex 2, France

S. Sanz

Departamento de Biología, Facultad de Ciencias,

Universidad Autónoma de Madrid, Madrid, Spain

H.-H. Steinbiss

Max Planck Institut für Züchturnsforschung,

Carl von Linne Weg 10, Cologne, Germany species, location or morphological features, transfer cells might differentiate in a similar way under the influence of conserved induction signals. In planta and yeast experiments showed that the promoter activity is modulated by carbohydrates, glucose being the most effective inducer.

Keywords Endosperm - Maize ·

Source-sink relationship · Transfer cells $\cdot$ Transport

$\begin{array}{ll}\text { Abbreviations } & \\ \text { BETL } & \text { Basal endosperm transfer cell layer } \\ \text { ESR } & \text { Embryo surrounding region } \\ \text { MU } & \text { 4-Methylumbelliferone } \\ \text { MUG } & \text { 4-Methylumbelliferyl } \beta \text {-D-glucuronide } \\ \text { MUG-a } & \text { 4-Methylumbelliferyl } \beta \text {-D-galactoside } \\ \text { pmol MU min } & -1 \\ \text { mg protein }^{-1} & \text { Picomoles of MU produced per minute } \\ \text { X-glucuronide } & \text { 5-Bromo-4-chloro-3-indolyl-beta-D-glu- } \\ & \text { curonic acid cyclohexylammonium salt } \\ \text { ZmMRP-1 } & \text { Zea mays Myb related protein 1 }\end{array}$

\section{Introduction}

In higher land plants, nutrients flow between the photosynthetically active tissues (known as sources) and the storage organs or the young parts of the plant (known as sinks); the growth of the latter depends on this supply (Williams et al. 2000). This flux takes place through phloem sieve elements, cells that are intimately connected to modified companion cells by numerous, branched plasmodesmata. The companion cells, which nurse and feed the sieve elements, are located in the loading and unloading areas of the vascular tissues, and often show typical transfer cell morphology characterized by a large number of cell wall ingrowths 
facing towards the sieve elements (Pate and Gunning 1972; Thompson et al. 2001; Offler et al. 2002). The development of such ingrowths has also been reported in the phloem parenchyma cells of the minor veins of Arabidopsis thaliana leaves (Haritatos et al. 2000).

Transfer cells are seen in all plant taxonomic groups, including algae, as well as in fungi (Pate and Gunning 1972). The secondary wall ingrowths of these cells can increase the plasma-membrane surface area by up to 20 -fold, thus enhancing its transport capacity. It has been proposed that transfer cells play significant roles in nutrient transport pathways. Indeed, these cells are strategically positioned at the interfaces where solute exchange takes place, for example, at leaf minor veins, at distribution areas of xylem and phloem sap in stem nodes, at sites of glandular secretion, at points of delivery of nutrients to sink organs (at the base of flowers and fruits), and at the symplastic discontinuities between individuals belonging either to different generations [i.e., at the base of seeds (Kiesselbach 1949; Cochrane and Duffus 1980; Ligrone and Gambardella 1988; Offler and Patrick 1993; Talbot et al. 2001)], or species [for example in symbiotic associations like mycorrhizal and rhizobium-root nodule interfaces (Gunning et al. 1974; Allaway et al. 1985) and plantparasite interactions like nematode infections (Jones and Northcote 1972)].

Developing seeds are a typical example of sink elements since they are net importers of organic and inorganic nutrients. Seeds synthesize starch from sucrose and sucrose-derived metabolites that they import from the maternal vascular system terminals. Since there are no symplastic connections between the maternal and filial cells, the solutes are apoplastically uploaded by the embryo and the endosperm. To promote solute uptake, in many species the outer cell layers of the storage organs (generally the endosperm in monocots and the cotyledons in dicots) differentiate into transfer cells in the region facing the maternal vascular terminals. In the maize kernel, at the base of the endosperm and adjacent to the pedicel, the aleurone layer is substituted by the transfer cell layer; this is specialized for nutrient uptake and transport from the maternal tissues to the developing endosperm (Thompson et al. 2001; Royo et al. 2007).

Transfer cell differentiation presumably occurs as a response to an increased demand for solute transport. Little is known about the molecular signals that induce transfer cell differentiation, but it is likely that transported solutes are involved in the process (Offler et al. 2002). We have recently obtained evidence (data not shown) indicating that ZmMRP-1 (Gómez et al. 2002) is a primary sensor of this signal in maize and that it induces transfer cell differentiation at the base of the maize endosperm.
ZmMRP-1 was the first transfer cell-specific transcriptional activator to be identified. The protein contains a 53amino acid domain highly homologous to the MYB-related DNA binding domain identified in several DNA binding proteins of the SHAQK(Y/F)F sub-family (MybSt1, LeMYB1, LHY and CCA1). The expression of ZmMRP-1 is readily detected in the basal part of the endosperm as early as 3 days after pollination (DAP) (when the endosperm coenocyte is still organized into nuclear-cytoplasmic domains) and continues during the development of transfer cells. ZmMRP-1 also regulates the expression of the transfer cell specific genes BETL-1, BETL-2 (Gómez et al. 2002), Meg-1 (Gutiérrez-Marcos et al. 2004) and ZmTCRR-1 (Muñiz et al. 2006).

In this work, a functional promoter of ZmMRP-1 was identified in maize. The introduction of this promoter into several heterologous species provided important information on the signals inducing the expression of ZmMRP-1 and, consequently, the differentiation of functional transfer cells. Two major causes inducing promoter activity were found to be: (1) the need to support active transport across developing exchange surfaces (such as in young branching areas) before proper vasculature connections are established; and (2) the increase in transport demand caused by the establishment of a strong sink, such as at the base of developing fruits and in nematode feeding structures. The present results also show that the promoter is modulated, in these sites, by the concentration of monosaccharides.

\section{Materials and methods}

Plasmid constructs

Plants were transformed using different methods with vectors containing $850 \mathrm{bp}$ of the proximal ZmMRP-1 promoter sequence (Gómez et al. 2002) fused to the GUS reporter gene.

For maize transformation, the fragment ZmMRP-1prom was obtained from the plasmid ZmMRPprom-GUS (Gómez et al. 2002) and cloned in a Gateway derived vector (Invitrogen) that contains the ZmMRP-1 promoter linked to GUS and an Arabidopsis Sac66 polyadenylation signal. The expression cassette was transferred into a GATEWAY Destination plant binary vector based on pSB12 (Komari et al. 1996). For barley transformation, the fragment ZmMRP-1prom-GUS-35Ster was obtained from the plasmid ZmMRPprom-GUS (Gómez et al. 2002) and cloned in the Agrobacterium binary vector pWBVec8 (Wang et al. 1998).

To prepare the Arabidopsis and tobacco reporter lines, a pBI101.3 $\Delta$ GUS Agrobacterium binary vector, derived 
from pBI101.3 (Clontech) was used to subclone the ZmMRP-1prom-GUS-35Ster cassette.

Plant transformations

Zea mays [cv. A188, obtained from the National Plant Germplasm System (NPGS) of the United States Department of Agriculture-Agricultural Research Service, USDAARS, http://www.ars-grin.gov], Hordeum vulgare (cv. Golden promise, also obtained from the USDA-ARS), Nicotiana tabacum (cv. Petit Havana line SR1, obtained from Japan Tobacco Co. Ltd, Japan) and Arabidopsis thaliana (ecotype Col-0, obtained from the Nottingham Arabidopsis Stock Centre (NASC), http://arabidopsis.info) were used to produce ZmMRP-1p-GUS reporter lines.

For maize transformation, the plasmid BIOS1190 was transferred to Agrobacterium tumefaciens LBA4404 (pSB1) according to Komari et al. 1996, and the maize transformed essentially as described by Ishida et al. (1996). Barley transgenic lines were generated by Agrobacterium tumefaciens (strain AGL1)-mediated transformation of immature embryos as described by Wang et al. 1998.

Maize and barley transgenic plants were analyzed by Southern blotting: DNA was extracted from young leaf material as described by Sambrook et al. (1989); $15 \mu \mathrm{g}$ of DNA was digested and blotted onto nylon membranes (Roche) hybridized with a ${ }^{32} \mathrm{P}$-radiolabelled probe containing the $3^{\prime}$ end of the GUS gene (Hueros et al. 1995).

Transgenic tobacco plants were obtained as described (Keil et al. 1989) from leaf disks of tissue cultured plants co-cultivated in a LBA4404 Agrobacterium tumefaciens suspension. Plant transformants were selected for resistance to kanamycin and, after rooting, were transferred to soil and grown in the greenhouse. The presence and integrity of the constructs was tested by Southern hybridization using $10 \mu \mathrm{g}$ of genomic DNA as described above.

One-month-old Arabidopsis plants were used for floral dip transformations mediated by C58C1pGV3101 Agrobacterium tumefaciens as described by Clough and Bent (1998). Seeds of primary transformants were plated on Murashige and Skoog (MS) medium containing $0.8 \%$ agar, $1 \%$ sucrose with $50 \mu \mathrm{g} / \mathrm{mL}$ kanamycin, and $50 \mu \mathrm{g} / \mathrm{mL}$ ampicillin. Resistant plants were transferred to soil after 2 weeks. Blot hybridization analysis of the nucleic acids was performed using $5 \mu \mathrm{g}$ of genomic DNA, as described above.

\section{GUS activity assays}

Expression of the GUS gene was detected by histochemical staining according to the method of Jefferson et al. (1987). Tissues were stained for GUS in a medium containing $0.5 \mathrm{mg} / \mathrm{mL} \mathrm{X}$-glucuronide (Duchefa), $0.5 \mathrm{mM} \mathrm{K}{ }^{+}$-ferrocya- nide, $0.5 \mathrm{mM} \mathrm{K}^{+}$-ferricyanide, $10 \mathrm{mM} \mathrm{Na}{ }_{2}$ EDTA, $50 \mathrm{mM}$ phosphate buffer, $\mathrm{pH} 7,0.1 \%$ Triton $\mathrm{X}-100$, and $20 \%(\mathrm{v} / \mathrm{v})$ methanol. Plant tissues were counterstained with Safranin$\mathrm{O}$ (Sigma) in $50 \% \mathrm{EtOH}$.

GUS activity quantitative assays were performed according to Jefferson et al. (1987). Pools of 150-200 seeds each were germinated for $36 \mathrm{~h}$ on solid MS medium containing glucose, sucrose, fructose or mannitol as the only sugar source at a concentration either 0.11 or $0.165 \mathrm{M}$. Three independent replicas were performed for each experiment. Seeds were homogenized in GUS extraction buffer (0.1 $\mathrm{M} \mathrm{Na}_{2} \mathrm{HPO}_{4}, \mathrm{pH} 7,0.25 \mathrm{M}$ EDTA, $0.1 \%$ Triton X-100 and $0.0007 \% \beta$-mercaptoethanol) and the protein concentration measured by the Bradford assay (Bradford 1976) using bovine serum albumin as a standard. 15-30 $\mu \mathrm{g}$ of total protein were used for the fluorimetric GUS expression assay using MUG (4-methylumbelliferyl $\beta$-D-glucuronide) as a substrate. Fluorescence of the MU (4-methylumbelliferone) product was measured using a Wallac Victor ${ }^{2} 1420$ multilabel counter plate reader (Perkin Elmer). The results are expressed as picomoles of MU produced per minute and milligram of total protein.

\section{Yeast assays}

Saccharomyces cerevisae strain MaV203 (Invitrogen) was transformed with vector pYC7 (Chang and Timberlake 1992) containing the $850 \mathrm{bp} Z m M R P-1$ promoter sequence fused to the lac $Z$ reporter gene. This plasmid confers yeast cells the ability to grow in an SD medium lacking uracil (SD-ura). Transformed cells were grown at $30^{\circ} \mathrm{C}$ in liquid SD-ura medium containing $2 \%$ glucose until an $\mathrm{OD}^{600}$ of 1 was obtained. Cells were collected by centrifugation, washed in sterile deionized water and divided into equal aliquots that were resuspended in SD-ura medium containing $2 \%$ glucose, $2 \%$ fructose, $2 \%$ sucrose or $2 \%$ mannitol. Three replicates of each culture were performed. After growing for $4 \mathrm{~h}$ at $30^{\circ} \mathrm{C}$, the cells were collected by centrifugation and total protein extracted using YeastBuster ${ }^{\mathrm{TM}}$ Protein Extraction Reagent (Novagen). Protein concentration was measured by the Bradford assay (Bradford 1976) using bovine serum albumin as a standard; $19 \mu \mathrm{g}$ of total protein was used in a standard fluorimetric assay of $\beta$-galactosidase activity using MUG-a (4-methylumbelliferyl $\beta$-D-galactoside) as a substrate. These assays were undertaken at $25^{\circ} \mathrm{C}$ in a final volume of $200 \mu \mathrm{l}$ of $60 \mathrm{mM}$

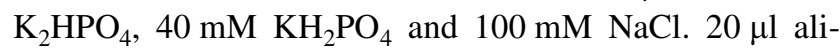
quots were withdrawn at $0,20,40,60$ and $80 \mathrm{~min}$. After stopping the reaction with $0.2 \mathrm{M} \mathrm{Na}_{2} \mathrm{CO}_{3}$, the fluorescence of the 4-methylumbelliferone (MU) produced by hydrolysis of MUG-a was measured using a Wallac Victor ${ }^{2} 1420$ instrument (Perkin Elmer) and compared with the fluorescence produced by known quantities of MU. The results are 
expressed as picomoles of MU produced per minute and milligram of total protein.

Histological analyses

Plant material was stained for GUS as describe above. Seed samples were then fixed with FAA (10\% acetic acid glacial, $5 \%$ formaldehyde, $50 \%$ ethanol) for $30 \mathrm{~min}$. After this treatment, the germinating seeds were dehydrated and stained for $24 \mathrm{~h}$ with $0.2 \%$ eosin in ethanol. The samples were then embedded in wax (Paraplast, Sigma) using tertbutanol as a solvent (Weigel and Glazebrook 2002).

Stems from tobacco were fixed in $4 \%$ paraformaldehyde, $0.1 \%$ glutaraldehyde in $0.1 \mathrm{M}$ sodium phosphate buffer $\mathrm{pH}$ 7.2 for $12 \mathrm{~h}$. Samples were dehydrated and embedded in wax (Paraplast, Sigma) using xylol as a solvent (Hueros et al. 1995).

Sections $8-10 \mu \mathrm{m}$ thick were fixed to glass slides treated with 3-aminopropyltriethoxylane, deparaffinated in xylol, counterstained with Safranin O, and mounted in DEPEX.

\section{Nematode infection}

The root-knot nematode, Meloidogyne javanica, was obtained from Dr. F Grundler (University of Natural Resources and Applied Life Sciences, Vienna, Austria) and maintained in our laboratory, growing in vitro on cucumber roots. Twelve to fifteen Arabidopsis seedlings were grown per plate for 8 days in a growth chamber at $22^{\circ} \mathrm{C}$ and under a 16-h light and 8-h dark photoperiod. Second stage nematode juveniles (J2) were then used to inoculate these Arabidopsis plants (using about 100 individuals per plant).

Infected roots were examined using a stereo microscope to detect the swelling corresponding to gall development. GUS activity was assayed at 5, 10, 15 and 20 days after nematode inoculation.

\section{Results}

Characterization of a functional promoter for ZmMRP-1

The isolation of ZmMRP-1 genomic clones has previously been reported (Gómez et al. 2002). An 850-bp fragment located upstream from the start codon of ZmMRP-1 was fused to the GUS reporter gene and the resulting construct introduced into the experimental maize plants. Of seven primary transformants analyzed, six showed GUS expression in the immature kernels. Detailed analysis of the GUS expression patterns in these plants revealed the tested 850bp fragment to be indeed a functional ZmMRP-1 promoter that drives GUS reporter expression exclusively in the endosperm transfer layer (Fig. 1). The GUS expression

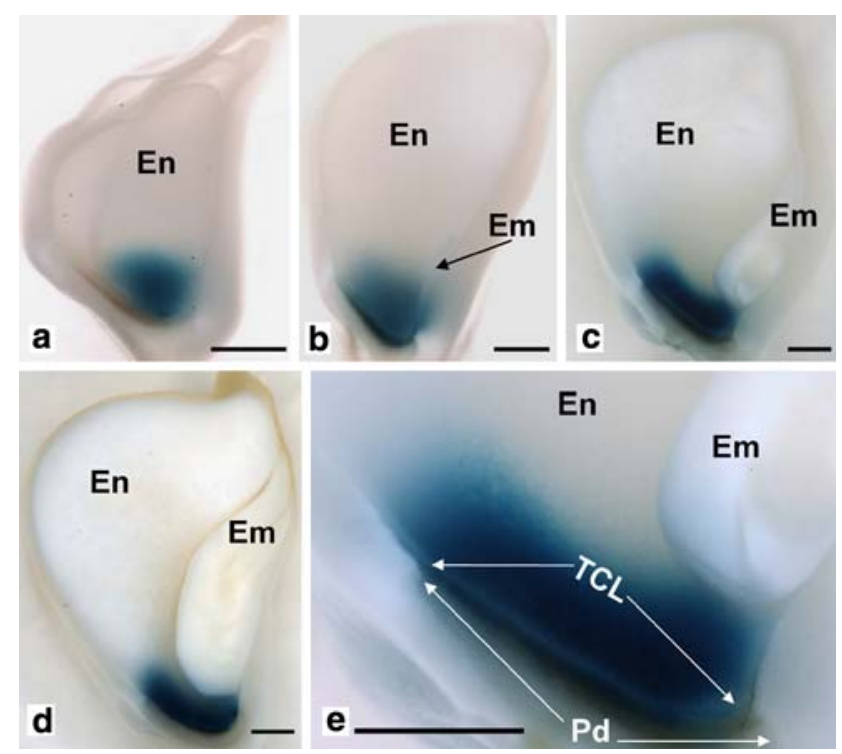

Fig. 1 a-e The promoter of $Z m M R P-1$ shows transfer cell specificity in maize. GUS staining pattern of ZmMRP-1prom-GUS transgenic kernels at 10 (a), 14 (b) 20 (c) and 30 (d) days after pollination (DAP). e Basal area of the endosperm from $\mathbf{c}$ at a higher magnification. An indigo precipitate, indicating GUS activity, is observed in the transfer cell layer $(T C L)$, which extends at the base of the endosperm, in front of the phloem terminals at the pedicel. En endosperm, Em embryo, $P d$ pedicel. Bars $1 \mathrm{~mm}$

pattern was compatible with the reported transcription pattern of the ZmMRP-1 gene in transfer cells (Gómez et al. 2002), although the timing of expression of the reporter seems to be slightly delayed as compared to the expression of ZmMRP-1. No signal was detected in other plant tissues, either in roots, stem, leaves or tassels. Within the endosperm, the expression of the reporter seems to be restricted to the basal transfer cell layer, although expression of the reporter gene in the embryo surrounding region, immediately adjacent to the transfer cell layer, can not be excluded from the histochemical analyses shown in Fig. 1.

\section{Promoter activity in barley}

Barley is a temperate cereal whose seeds contain the same basic components present in maize, but in a clearly different spatial arrangement. Consequently, the question arises as to whether, in this species, the functional ZmMRP-1 promoter targets tissues functionally equivalent to the maize transfer cells. In barley, nutrients pass symplastically via sieve elements through the chalazal region to the maternal nucellar tissues at the crease, and from there to the endosperm cavity, which runs parallel to the phloem strands along the length of the grain. The outer cell layer of the endosperm, which is in intimate contact with the cavity, is also modified to form a transfer cell layer, the functional homologue 
of the maize endosperm transfer cell layer (Thompson et al. 2001; Royo et al. 2007).

Thirty-six barley transgenic lines containing the ZmMRP-1pGUS construct were analyzed. The GUS signal was detected in developing grains in the endosperm transfer cells (also referred to as the modified aleurone layer) surrounding the endosperm cavity (Fig. 2c).

Unlike in maize, in which the ZmMRP-1 promoter is only active in the endosperm transfer cell layer, in the barley transgenic plants a strong GUS signal was also detected at the base of the spikelets (Fig. 2a, b) where the vascular tissues within the plexus contain numerous highly differentiated xylem and phloem transfer cells. Each sterile glume possesses vascular bundles that contain transfer cells only near the region where the glume is attached to the rachis (Zee and O'Brien 1971), the tissue where ZmMRP-1p-GUS activity was observed. No signal was found in other barley organs or tissues (data not shown).

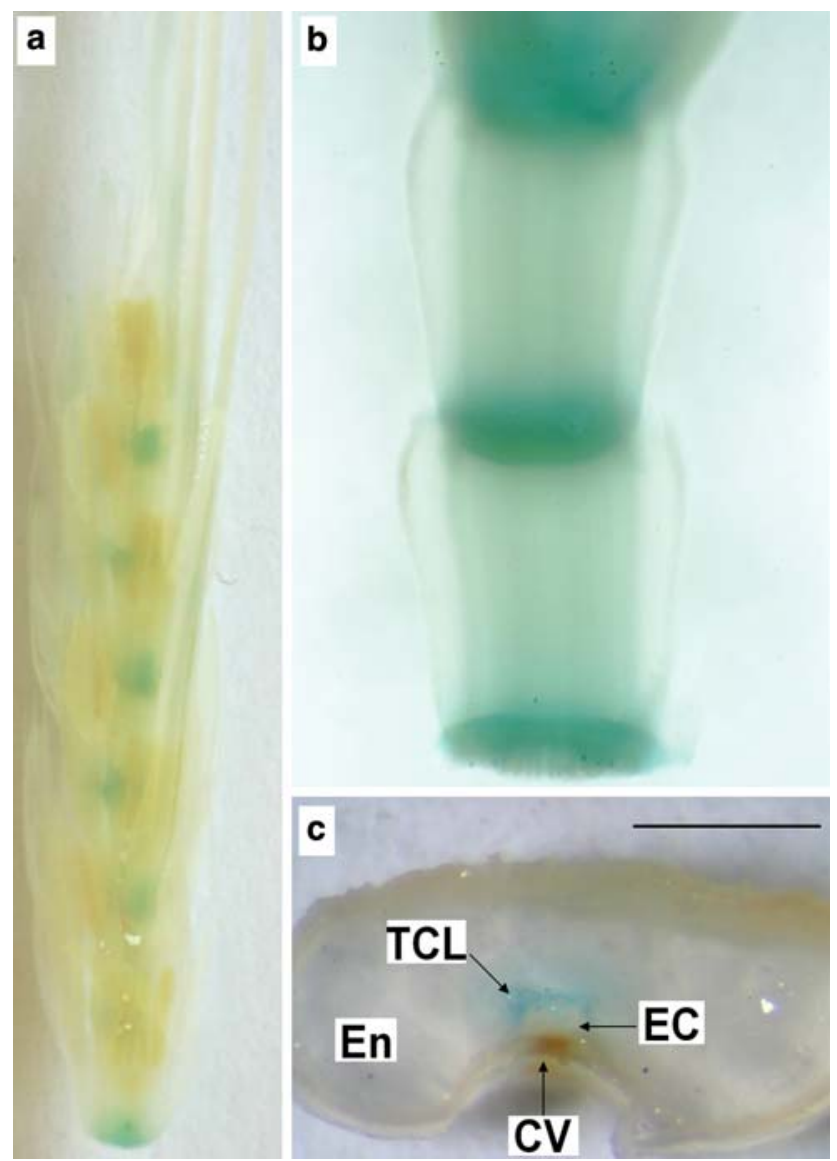

Fig. 2 a-c $Z m M R P-1$ promoter activity in barley. a GUS staining of an immature barley spike collected at the time of booting. b Detailed view of the rachis after removal of the spikelets. $\mathbf{c}$ Cross section of a 5-10 DAP kernel showing GUS signals in the endosperm transfer cell layer (TCL). EC endosperm cavity, $C V$ central vein, En Endosperm. Bar $1 \mathrm{~mm}$
Promoter activity during seed germination of dicots

Dicot seeds, particularly those of non-endospermic species, have a very different structure to cereal seeds, even though an ephemeral endosperm has been described in tobacco and Arabidopsis. The organization of the posterior endosperm chalazal cyst and its anatomic relationship with vascular terminals suggest that this tissue might be a functional homologue of the BETL, but a role for the posterior endosperm and maternal tissues in transporting nutrients remains to be demonstrated (Berger 2003; Olsen 2004). In this work, eight lines of tobacco and six of Arabidopsis transgenic plants containing the ZmMRP-1pGUS construct were obtained, the GUS expression pattern described below was identical in all the lines, although the signal intensity varied among lines (not shown), probably reflecting chromosomal position effects on the construct activity. In contrast to the situation observed in cereals, no promoter activity could be detected in either Arabidopsis or tobacco developing seeds (data not shown), a strong GUS signal was detected, however, in seeds germinated in MS medium containing $1 \%$ sucrose. In germinating tobacco seeds, GUS activity was mainly detected at the micropylar region (also referred to as the endosperm cap) of the endosperm, through which the radicle emerges at germination (Fig. 3a, b). A weaker GUS signal was also detected later in development in the peripheral endosperm, the functional equivalent to the cereal aleurone layer (data not shown, Olsen 2004).

In transgenic Arabidopsis seeds, the GUS expression pattern essentially reproduced that seen in tobacco. This signal was first detected at the micropylar region at the time of radicle emergence (Fig. 3e, f) and later on in the peripheral cells surrounding the embryo (Fig. 3c, d). Note that the plant had nearly completely (Fig. 3c) or completely (Fig. 3d) emerged from the seed coat-endosperm in these older seedlings, whilst it was completely included in the seed coat in the other images presented in Fig. 3. The peripheral endosperm has been related to the aleurone layer of cereals since both produce hydrolases, glucanases and proteinases when germination starts (Bethke et al. 2007). The signal intensity in the peripheral cells increased when the seeds were germinated in liquid media containing increasing concentrations of glucose (not shown).

Promoter activity in the nodal regions of seedlings and young branching points of adult plants

Strong ZmMRP-1 promoter activity was detected in the intercotyledonary regions of the stems of young tobacco and Arabidopsis seedlings (Fig. 4). The intensity of the signal decreased as the seedling matured, and finally disappeared when the leaves are fully developed (not shown). 


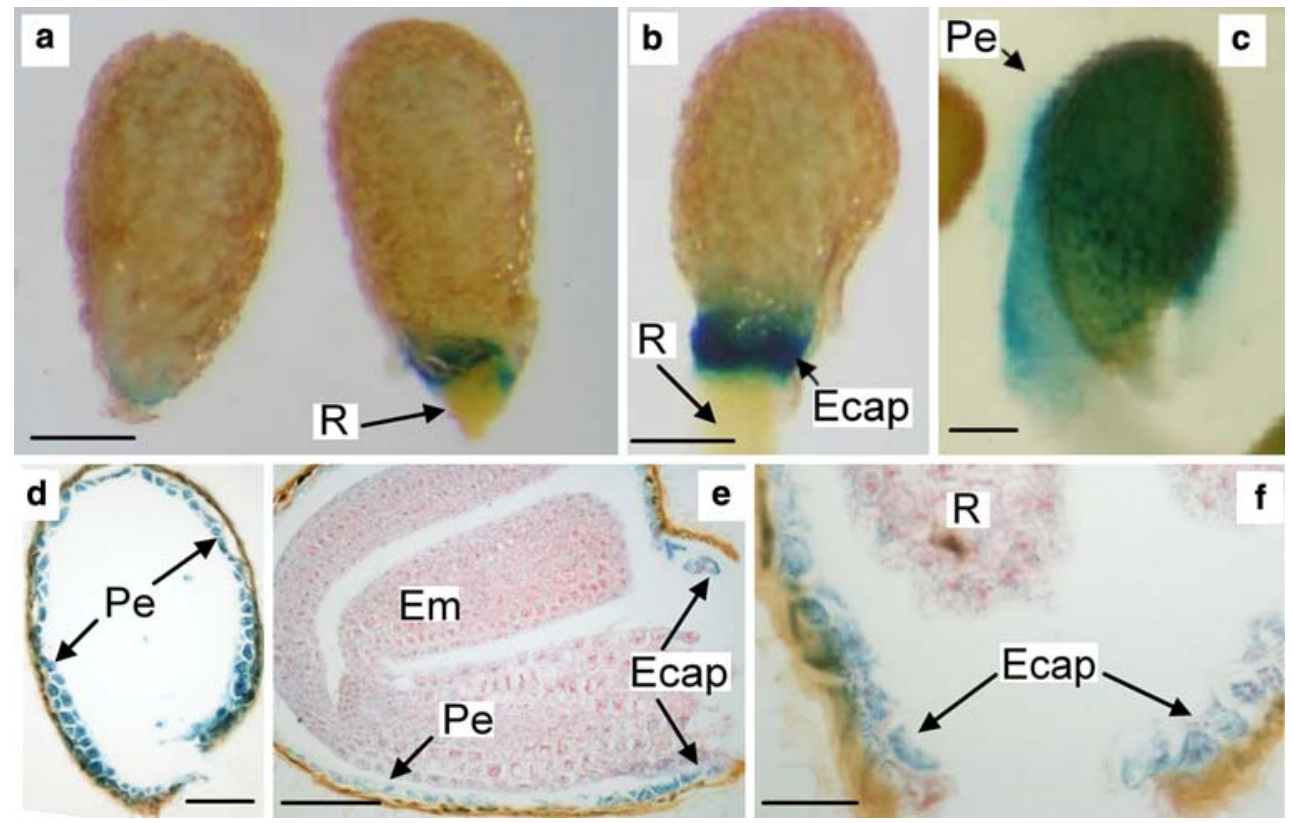

Fig. 3 a-f At germination, the promoter of $Z m M R P-1$ is active in the endosperm of transgenic Arabidopsis and tobacco. a and $\mathbf{b}$ Germinating tobacco seeds stained for GUS; a faint signal is observed over all the endosperm but it is particularly intense in the micropylar endosperm or endosperm cap (Ecap); three seeds showing progressively more developed radicle $(R)$ are shown. c-f Germinating Arabidopsis seeds. c GUS activity is patent in the peripheral endosperm $(\mathrm{Pe})$ in older seedlings. d Transversal section of a seed coat and peripheral endosperm once the plantlet has emerged. e Longitudinal section of a germinating seed. Em embryo. f Enlarged view of the micropylar endosperm (Ecap). Bars $1 \mathrm{~mm}(\mathbf{a}, \mathbf{b}), 100 \mu \mathrm{m}(\mathbf{c}-\mathbf{f})$

from transgenic Arabidopsis seeds germinated for $36 \mathrm{~h}$ on solid medium containing glucose, fructose, sucrose or mannitol (Fig. 4e). GUS activity depended on the sugar included in the medium, glucose being the best inducer at the two concentrations tested, 0.11 and $0.165 \mathrm{M}$. GUS activity in the presence of glucose increased from $1,267 \pm 199$ to $1,583 \pm 245 \mathrm{pmol} \mathrm{MU} \mathrm{min}{ }^{-1} \mathrm{mg}_{\text {protein }}{ }^{-1}$, whereas in the presence of the non-metabolizable sugar mannitol the corresponding values were $629 \pm 190$ and $1,093 \pm 45$ pmol MU min $^{-1} \mathrm{mg}$ protein $^{-1}$. GUS activity quantification was not possible in protein extracts from plantlets sown in media containing different sugars, a very low GUS activity was measured in all cases (not shown) possibly reflecting the relative low number of cells that express GUS in each plantlet.

These experiments did not show, however, which sugar ultimately arrives at the cells expressing the construct (the site of expression remains unchanged in all treatments). In addition, the possibility remains that the sugar promoter-inducing activities were the result of its effect on the plant physiology, for instance, at the level of transport rate, rather than a more direct effect on the promoter transcriptional activity. To overcome these difficulties, the effect of the different sugars on ZmMRP-1 promoter activity was analyzed in Saccharomyces cerevisae cells carrying a construct containing the 850-bp promoter fused to the lacZ reporter gene. To minimize 

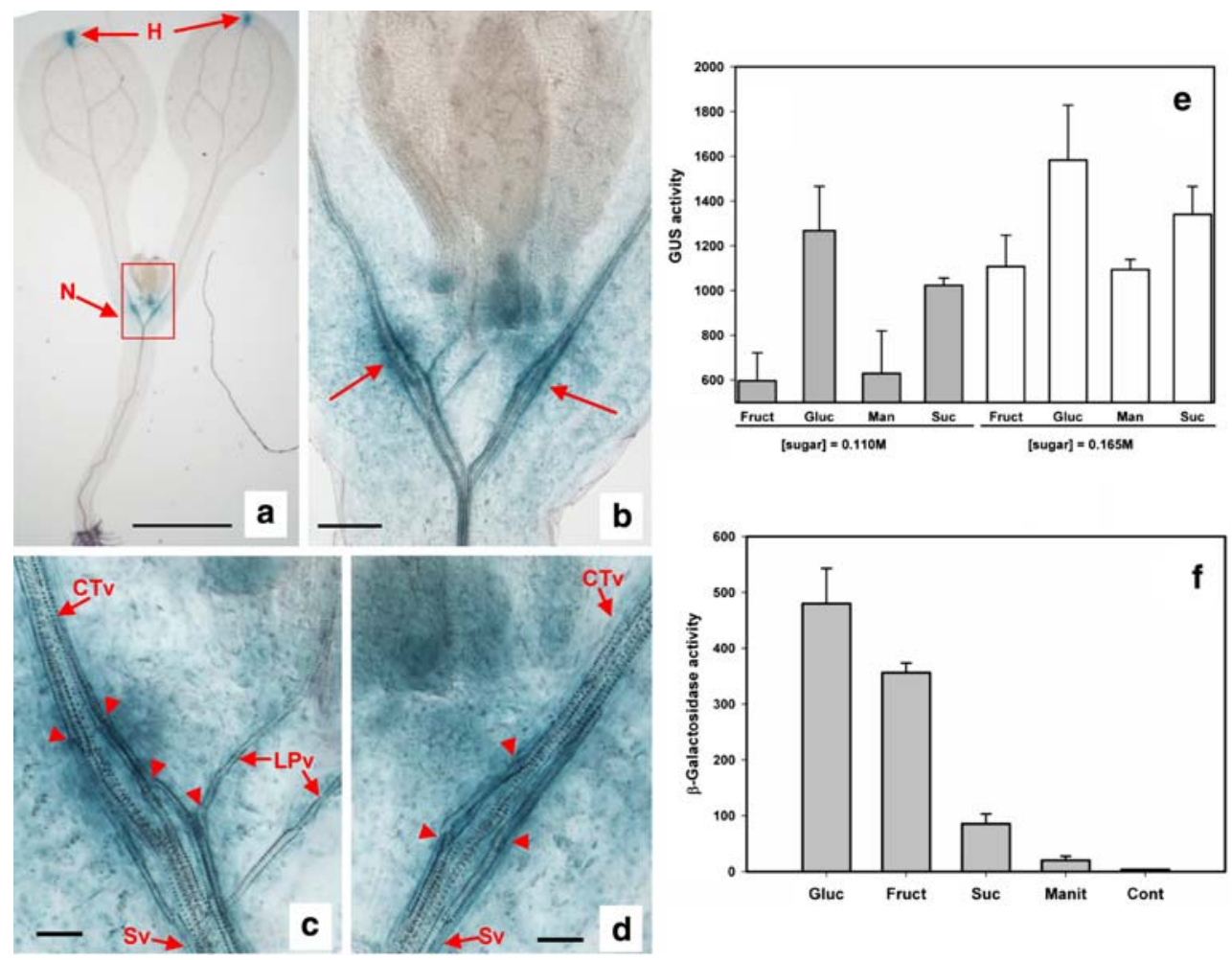

Fig. 4 a-f The ZmMRP-1 promoter is regulated by sugars in Arabidopsis seedlings and yeast. GUS activity (a-d) was detected in the intercotyledonary nodes $(N)$ and hydathodes $(H)$ in 7-10 day-old transgenic plantlets. The nodes area, squared off in red in a, is shown at higher magnification in b. Arrows point to the vascular nodes formed at both sides of the shoot apical meristem. The left and right nodes are shown at higher magnification in $\mathbf{c}$ and $\mathbf{d}$, respectively. Arrowheads in $\mathbf{c}$ and d point to the vascular branching and merging areas that concentrate most of the GUS signal. CTv cotyledon vasculature, $S v$ stem vasculature, $L P v$ leaf primordia vasculature. Bars $1 \mathrm{~mm}(\mathbf{a}), 100 \mu \mathrm{m}(\mathbf{b})$,

the effect of the physiological stage of the cells on promoter activity, all yeast cells were grown to logarithmic phase on standard SD medium containing $2 \%$ glucose before being incubated in SD medium with glucose, fructose, sucrose or mannitol as the only sugar source. Promoter induction, measured as $\beta$-galactosidase activity $4 \mathrm{~h}$ later, was strongly dependent on the sugar source (Fig. 4f); the promoter was moderately activated by sucrose and strongly induced by the monosaccharides of sucrose (especially glucose).

GUS activity was detected with similar patterns in Arabidopsis and tobacco, at the branching points of young stems in adult plants (Fig. 5). In these regions, the xylem parenchyma cells of the departing foliar traces were transformed into transfer cells (Fig. 5c, d; Gunning et al. 1970). GUS signal intensity reached a maximum when a new branch, leaf or reproductive structure started to depart from the stem. Subsequently, it faded away as these lateral structures developed their vascular connections with the main vascular system of the stem.
$25 \mu \mathrm{m}(\mathbf{c}, \mathbf{d})$. e Transgenic Arabidopsis seedlings (about 200 seeds per assay) were germinated in media containing the indicated concentrations of sugars. GUS activity was measured in the crude protein extracts. At the two sugar concentrations tested, glucose and sucrose (to a lesser extent) induced the promoter. f Promoter activity was measured in yeast clones containing a $Z m M R P-1$ promoter-lac $Z$ construct. Reporter gene activity was measured after incubation of the cells in media containing, as indicated, glucose, fructose, sucrose, mannitol or glycerol (cont). Glucose and fructose strongly induced the promoter. Mean values $\pm \operatorname{SD}(\mathbf{e}, \mathbf{f})$

Promoter activity at the base of reproductive organs

In Arabidopsis plants, GUS activity was detectable at the base of the fruits. The intensity of the signal was correlated with the developmental stage of these fruits, and consequently their strength as sinks. The maximum activity of the ZmMRP-1 promoter was observed at the first stages of fruit development, when nutrient uptake was highest in the immature siliques; it then decreased to become almost undetectable at full maturity (Fig. 6a).

In tobacco plants, this signal was also detected at the base of the flowers and fruits (Fig. 6b, c). At the base of the tobacco capsule, the GUS signal was located bordering the vascular system, from where it extended to the "placenta" formed by the phloem terminals at the base of the seeds (Fig. 6c).

The existence of a positive correlation between the activity of the ZmMRP-1 promoter and sink strength was confirmed in the tobacco plants, where the GUS signal disappeared at the base of the capsule of a non-pollinated 

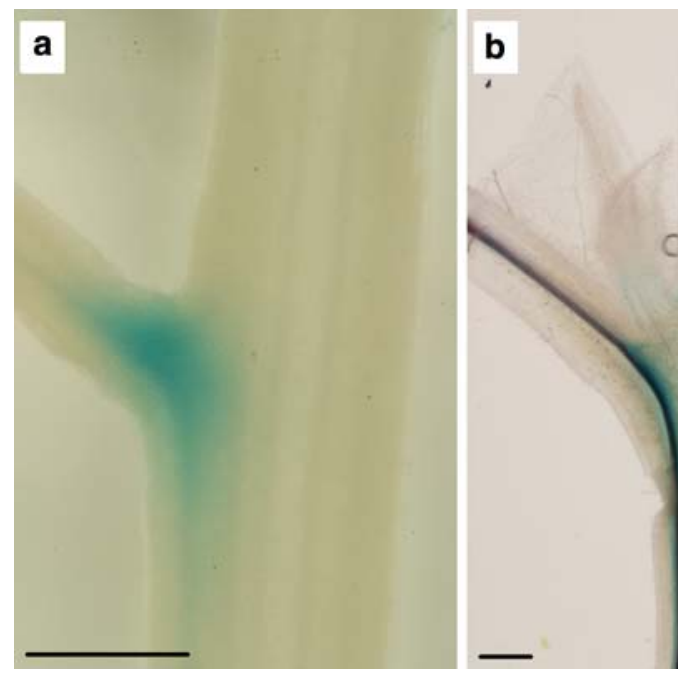

Fig. 5 a-d Expression of $Z m M R P-1$ at the young branching points of mature plants. a A branching point in the stem of Arabidopsis; the GUS signal is concentrated on the dorsal side of the vasculature. b Mid section of a tobacco stem at the emerging point of two lateral leaves and branches; areas squared off in red in $\mathbf{b}$ and $\mathbf{c}$ indicate the regions used

flower while normal pedicel staining persisted at the base of a pollinated flower on the same branch (Fig. 6d).

Promoter activity in giant cells induced by nematodes

Nematode infection leads to the development of feeding structures, and therefore sinks, containing cells with transfer cell features (Jones and Northcote 1972). The GUS expression signal driven by the ZmMRP-1 promoter was detected during the first stages of Arabidopsis root infection by Meleidogyne javanica. During this type of infection, some xylem parenchymatous cells are selected for the development of a feeding structure formed of multinucleate giant cells. These cells suffer synchronous nuclear divisions without cytokinesis, the development of cell wall invaginations and hypertrophy. Galling of the cortical tissue enclosing the feeding site and the growing nematode accompanies these processes (Sijmons 1993).

ZmMRP-1 promoter activity was restricted to the period between 5 and 10 days after nematode inoculation (Fig. 7a, b); the first stage of gall development when the giant cells begin to establish the nutrient uptake process. Subsequently, the GUS signal decreased to become undetectable until the egg mass was secreted within a gelatinous matrix (Fig. 7c, d).

\section{Discussion}

The present results show that the proximal 850-bp fragment upstream of the start codon of ZmMRP-1 is sufficient to direct GUS reporter gene activity in several maize transgenic lines, and in a way that closely resembles the spatial and temporal pattern of ZmMRP-1 expression (Fig. 1; Gómez et al. 2002). The specificity of the ZmMRP-1 promoter was identical to that of other transfer cell promoters such as BETL-1 (Hueros et al. 1999a) and Meg-1 (Gutiérrez-Marcos et al. 2004). The expression of the reporter gene seems to be, however, delayed as compared to the endogenous gene, which is detected by in situ hybridization as early as 3 DAP and reaches a maximum of expression between 11 and 16 DAP (Gómez et al. 2002). This behavior was already observed for the promoter of BETL-1 (Hueros et al. 1999a) and could be interpreted as the result of (1) the low sensitivity of the GUS histochemical assay, which would make difficult the detection of the expression at the earliest developmental stages (2) the stability of the GUS protein, which would extend the duration of the apparent expression peak once the protein is accumulated. ZmMRP-1 is not expressed in the embryo surrounding region (ESR; Gómez et al. 2002), which constitutes a separate expression domain within the endosperm (OpsahlFerstadt et al. 1997). Activity of the ZmMRP-1 promoter in the embryo surrounding region cannot be excluded from our data; however, due to the close proximity of the ESR cells to the transfer cell layer at its ad-germinal side and the diffuse GUS signal that covers the entire basal endosperm (Fig. 1). Closer inspection of sectioned material (not shown) suggest that indeed the promoter activity is excluded from the ESR, but a precise mapping of the promoter activity would probably require in situ detection of GUS mRNA. 


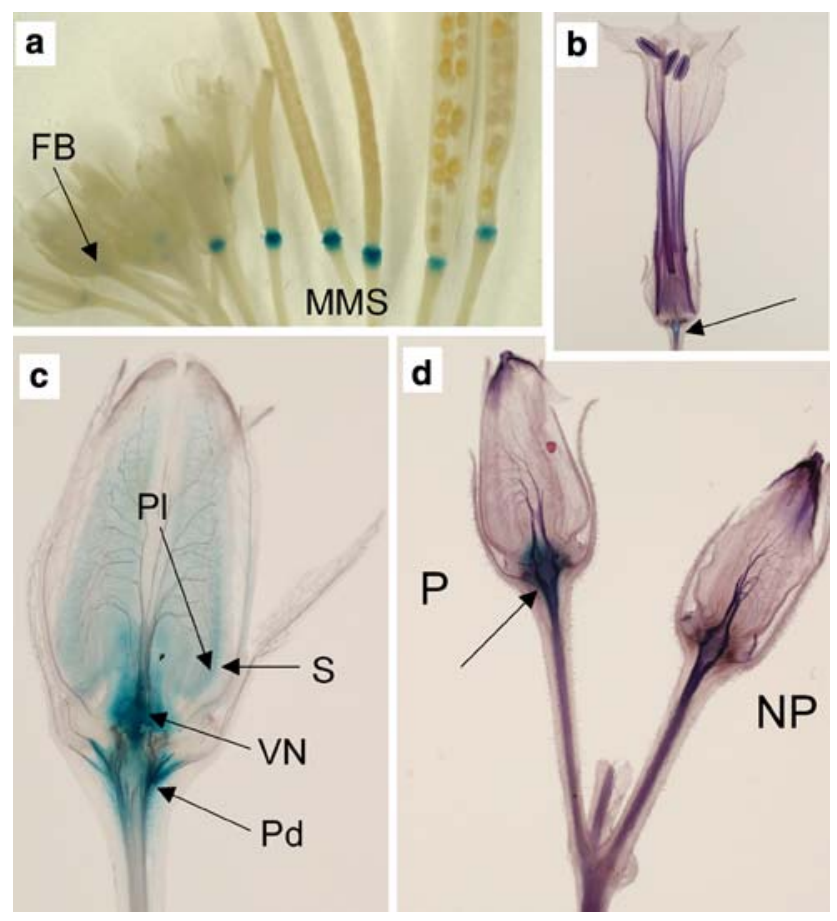

Fig. 6 a-d The $Z m M R P-1$ promoter is active at the base of reproductive structures. a GUS expression at the base of flowers and fruits in Arabidopsis; the signal intensity increases from flower budding $(F B)$ to reach a maximum at the pedicel of the siliques at the mid-maturation stage $(M M S)$; it then decreases as the fruit enters the seed filling phase. b GUS signal at the base of a tobacco flower (arrow). c The signal is most intense at the pedicel $(P d)$, the vasculature nodes $(V N)$ and the placental area $(P l)$ under the seeds $(S)$ of a pollinated tobacco flower at mid-maturation phase. d The GUS signal disappears from the pedicel of an emasculated non-pollinated flower $(N P)$ but reaches normal levels (arrow) in an emasculated manually-pollinated flower $(P)$ emerging at the same branching point

Identifying the signals controlling the activity of the ZmMRP-1 promoter in maize is impeded by the difficulty of accessing the endosperm transfer cells in order to manipulate the putative signals that diffuse from the maternal phloem cells (and which are presumably necessary for its differentiation) (Gómez et al. 2002). In previous studies, we were unable to detect or induce ZmMRP-1, or any other transfer cell-specific gene (Hueros et al. 1995, 1999a, b; Gómez et al. 2002), in maize plant tissues other than the endosperm transfer cells. This suggests that the mechanisms regulating gene expression in transfer cells are tightly controlled in maize. We, therefore, decided to introduce the ZmMRP-1prom-GUS construct into the heterologous species barley, Arabidopsis and tobacco, in which the promoter would likely be less strongly controlled and the resulting GUS expression patterns likely to provide additional information regarding the regulation of ZmMRP-1 promoter.

In temperate cereals, such as barley and wheat, seed anatomy strongly differs from that observed in maize (Royo
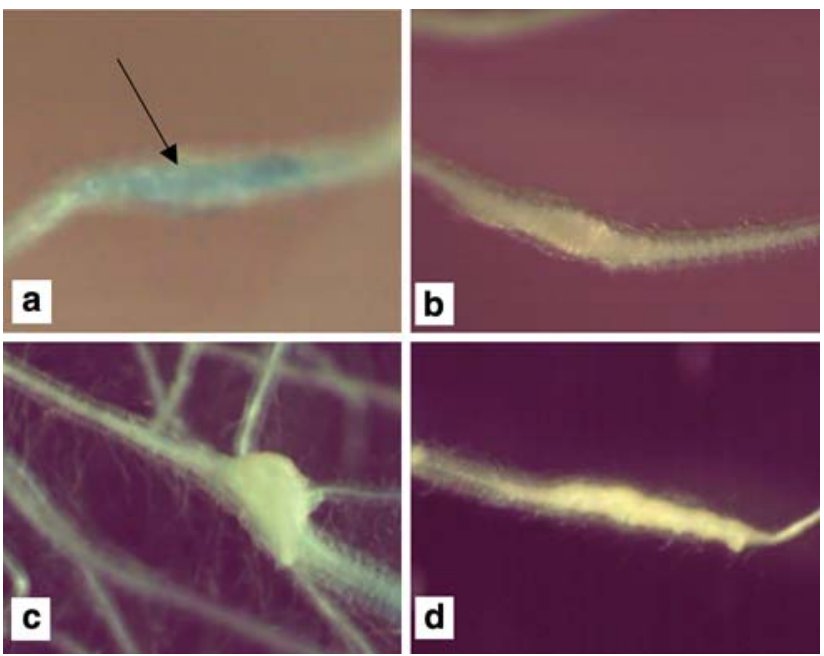

Fig. 7 a-d The promoter of $Z m M R P-1$ is active in the early developmental stages of the feeding structures induced by nematode infection. a A transgenic Arabidopsis root 5 days after inoculation with Meloidogyne javanica larvae showing the GUS signal in the gall (arrow). b A gall 10 days after inoculation. c A gall 15 days after inoculation. d A gall 20 days after inoculation

et al. 2007). Nutrients are uploaded into the endosperm from a cavity running along the seed, rather than from the pedicel as in maize. The endosperm cavity is surrounded by a modified aleurone layer composed of cells showing transfer cell morphology, and which are involved in the transport of nutrients into the developing endosperm (Wang and Fisher 1994; Weschke et al. 2003). In this work, ZmMRP-1 promoter activity was detected in the developing modified aleurone layer of the barley (Fig. 2c). This provides a functional link between the modified aleurone layer in barley and maize transfer cells, as well as a first indication that the promoter responds to functional, transport-related signals rather than positional signals, which would have targeted the promoter expression to the base of the barley grain. Interestingly, no expression was detected in the transfer cells found at the nucellar projection. This is consistent with the pattern of other maize transfer cell-specific promoters examined in barley (data not shown). In addition, the existence of endosperm and nucellar transfer cells as two separate expression domains has been demonstrated by the identification of genes specifically expressed in each of these tissues (Drea et al. 2005). The signalling mechanisms operating in the endosperm maize and wheat/barley transfer cells probably differ profoundly from those found at the nucellar projection transfer cells of wheat/barley. The former cell types are specialized in the uptake of nutrients to nurse sink organs (such as the developing endosperm or young leaves and stem branches; see below), while the latter facilitate the transit of nutrients from the vascular terminals to the endosperm cavity. GUS expression was also detected in the developing barley spikelets (see below). 
The expression pattern of the reporter gene in Arabidopsis and tobacco was basically identical, indicating that the signals regulating the in vivo expression of $Z m M R P-1$, and very likely the differentiation of transfer cell areas, are conserved in both species. GUS expression was detected in several tissues and organs in these transgenic reporter lines. The different patterns of expression can be assigned to two functional classes: (1) that of the nodal regions of young stems and the base of reproductive structures, and (2) that of the endosperm in germinating Arabidopsis and tobacco.

GUS expression in the nodal regions of young stems and at the base of reproductive structures

The GUS expression observed at different points of the vegetative structures in the present transgenic Arabidopsis and tobacco plants was always restricted to immature tissues apparently involved in the active transport of nutrients. In young tobacco and Arabidopsis seedlings, the expression of GUS was detected in the nodal region of the stems, at the base of the cotyledons, and at the end point of the cotyledonary leaves close to the hydathodes (Fig. 4). The presence of a "collar" of transfer cells at the cotyledonary nodes was described by Pate et al. (1970). They appear after seed germination and usually differentiate from the xylem parenchyma of the cotyledonary traces. These transfer cells play a very important role during the early nutrition of the young plumule, particularly before it develops proper vascular connections with the primary vascular system already connecting the cotyledons and roots (Pate et al. 1970; Pate and Gunning 1972). The GUS signal begins to decrease, and eventually disappears in the cotyledonary node when these vascular connections are finally established. The whole mount-high resolution images shown in Fig. 4b-d revealed that the promoter driven GUS expression reached a maximum at the anastomosis regions formed at the place where the vascular bundles from cotyledons, leaf primordia and stem converge. This further reinforces the suggestion that the ZmMRP-1 promoter responds to situations in which an active transport system is being established.

Hydathodes retrieve ions and organic molecules from the apoplasm and the xylem sap and exude water via the guttation stream; they are also involved in the selective absorption and retrieval of both inorganic and organic solutes (Esau 1977). In some species, cells positioned between the xylem and the hydathodes develop the characteristic transfer cell wall ingrowths, as they are the main regulators of the bidirectional transport established between the vascular tissue and the external environment (Pate and Gunning 1972). Again, the GUS signal was detected only in the cotyledons and young leaves, progressively decreasing (until disappearing) as they matured, even though hydath- odes continue to be functional in adult leaves. Therefore, as in maize and barley endosperm transfer cells, ZmMRP-1 promoter activity in solute exchanging areas is only detectable during the first stages of differentiation of the transport areas, becoming silent when transport ceases or the vascular connections are fully established.

In the nodes along the stem, the expression of GUS was also transitory and restricted to transport areas (Fig. 5). Transfer cells have been detected in the vascular tissues of nodes and adjacent region of internodes in a wide variety of plants (Gunning et al. 1970). In every case, these cells occur as a modified xylem parenchyma and are predominantly associated with departing foliar traces. These cells facilitate the transfer of nutrients to organs, like at the young apices and axillary meristems still not connected to the main vasculature. It should be noted that the expression of GUS in Arabidopsis and tobacco plants reproduces this pattern of development, with the GUS signal being restricted to the young nodes (Fig. 5a, b), and more specifically to the transfer cells modified from the xylem parenchyma (Fig. 5c, d). A similar situation has been reported in the spikelets of wheat, at the nodes where the sterile glumes are attached to the rachilla (Zee and O'Brien 1971). In the present barley reporter lines, GUS expression followed the development and differentiation of transfer cells in the vascular system, in the main bundles supplying nutrients to those floral organs (Fig. 2a, b).

The results of the analyses of GUS expression in developing flowers and fruits provide additional support to the hypothesis that the ZmMRP-1 promoter responds to the same signals that induce the differentiation of transfer cells. The intensity of the GUS signal at the base of the siliques increases over fruit development, reaching a maximum when the siliques are fully expanded, right before the fruits switch from the differentiation to the seed filling and maturation processes (Fig. 6a).

The interdependence between transfer cell differentiation and the need to establish an efficient transport mechanism has been investigated by experimental elimination of the sinks; fruit elimination or preventing fertilization abolishes the differentiation process (Pate and Gunning 1972). Similarly, in the present transgenic tobacco plants, the GUS signal disappeared from the pedicel of flowers in which pollination was prevented, but not from pollinated flowers on the same branch (Fig. 6d). Additional evidence of sink dependence on promoter activity was provided by the experimental infection of transgenic roots with nematodes (Fig. 7). The promoter of $Z m M R P-1$ was transiently active during infection when the feeding structures were being induced by the nematodes. No promoter activity was detected during the most active transport phase, when the giant cells have completely differentiated (Goverse et al. 2000). 
Promoter activity in the endosperm of germinating Arabidopsis and tobacco seeds

The expression of the ZmMRP-1 promoter in transgenic Arabidopsis and tobacco seeds at germination was an unexpected result. In cereal seeds, this promoter activity is strictly confined to the seed development phase (Gómez et al. 2002; this work). The lack of signal during the maturation phase agrees with the minimal contribution of the endosperm to seed development in dicot species, where it is consumed by the cotyledons and becomes a residual structure in mature seeds. This also suggests that the endosperm does not develop specialized transport structures and probably does not make a major contribution to the seed filling process, despite the apparently conserved antero-posterior organization of endosperm domains in cereals and dicots suggesting the existence of homologies between the posterior chalazal endosperm and the maize basal transfer cell layer (Berger 2003; Olsen 2004).

At germination, however, GUS expression was detected in the remnants of the peripheral endosperm, along with strong expression in the micropylar endosperm in germinating Arabidopsis and tobacco seeds (Fig. 3). The activation of the ZmMRP-1 promoter at seed germination appears not to be related to the development of transport structures. This activation might be caused by signals present in the promoter due to its endospermic origin; these signals would be read incorrectly in the dicot systems since the activity is detected in germinating, rather than developing seeds. Significantly, neither the maize nor barley transgenic plants showed any promoter expression in the germinating seeds (not shown). Alternatively, the induction of the ZmMRP-1 promoter in the endosperm of Arabidopsis and tobacco during germination might indicate that it is responsive to signals involved in defence responses. This would agree with the role of ZmMRP-1 as a transcriptional regulator of some genes encoding antimicrobial peptides, and with the putative defence role of the cereal endosperm transfer cells in developing grains (Serna et al. 2001). The presence of active defence-related signalling pathways in germinating seeds is not unexpected, in fact it has been suggested that the main function of the genes expressed by the endosperm during germination is to provide a prophylactic mechanism against microbial infection (Wu et al. 2001).

Sugars and the regulation of the ZmMRP-1 promoter

The observed increase in promoter activity when incubating germinating seeds and seedlings in increasing concentrations of sugars (Fig. 4e for quantitative data on germinating seeds) suggests that the increase in monosaccharides at the exchange areas might trigger the differentiation of transfer tissues. These results also confirm that cell identity is a major deter- minant of ZmMRP-1 expression, since the increase in reporter gene expression induced by glucose did not result in expression of the promoter at additional sites within the plant (not shown). These results suggest that developmental signals, rather than the concentration of any given metabolite, ultimately determine the sites of expression of ZmMRP-1. The concentration of sugars would then act as a modulator of the promoter activity; this effect is, however, difficult to study in the plant system since the nature of the signal that ultimately reaches the cells expressing the construct is unknown. Furthermore, the sugar composition of the different media might influence the physiology of the seedlings (including the transport rate), thus indirectly influencing promoter activity.

These difficulties are overcome in a cell system such as yeast, where the cells are grown up to log phase in identical media followed by a short incubation period with the different sugars. Indeed, the present results suggest a strong inductive effect of glucose and fructose on the ZmMRP-1 promoter in yeast (Fig. 4f).

In vitro experiments have shown a direct influence of sugars on transfer cell differentiation at the surface of Vicia faba cotyledons (Offler et al. 1997; Weber et al. 1997; Farley et al. 2000). These experiments indicate that apoplastic sugars, although more precisely the hexoses/sucrose ratio, induce and modulate epidermal transfer cell differentiation. Importantly, the present data show that the promoter of ZmMRP-1 regulates the site of expression of this transfer cell-specific transcriptional activator in response to tissuespecific signals, while the expression level at those sites is modulated by the concentration of hexoses.

This work shows that the ZmMRP-1 promoter is induced in developing transport-related cells located at the exchanging interfaces between different plant compartments. The promoter is silenced, however, when (1) a proper vasculature connection is established, thus making transfer cellfacilitated transport unnecessary, or (2) the transfer cells are fully differentiated and an active transport function, but no further cell differentiation, is required. The present results suggest that the ZmMRP-1 promoter might be induced by some of the solutes being transported.

Acknowledgments We thank the Cereal Functional Analysis Group (FAC) at Biogemma SAS, headed by Dr. Pascual Pérez, and the FAC maize transformation group headed by Dr. Denise Gerentes for their help. We also thank Y. Sanz for technical support. This work was supported by: Spanish Ministerio de Educación y Ciencia (BIO200303721 and BIO2006-14190 to G.H.); Biogemma SAS; Spanish Ministerio de Educación y Ciencia (Programme "Ramón y Cajal" to J.R.).

\section{References}

Allaway WG, Carpenter JL, Ashford AE (1985) Amplification of intersymbiont surface by root epidermal transfer cells in the Pisonia mycorrhiza. Protoplasma 128:227-231 
Berger F (2003) Endosperm: the crossroad of seed development. Curr Opin Plant Biol 6:42-50

Bethke PC, Libourel IGL, Aoyama N, Chung Y-Y, Still DW, Jones RL (2007) The Arabidopsis aleurone layer responds to nitric oxide, gibberellin, and abscisic acid and is sufficient and necessary for seed dormancy. Plant Physiol 143:1173-1188

Bradford MM (1976) A rapid and sensitive method for the quantitation of microgram quantities of protein utilizing the principle of protein-dye binding. Anal Biochem 7:248-254

Chang YC, Timberlake WE (1992) Identification of Aspergillus brZA response elements (BREs) by genetic selection in yeast. Genetics 133:29-38

Clough SJ, Bent AF (1998) Floral dip: a simplified method for Agrobacterium-mediated transformation of Arabidopsis thaliana. Plant J 16:735-743

Cochrane MP, Duffus CM (1980) The nucellar projection and modified aleurone in the crease region of developing caryopses of barley (Hordeum vulgare L. var. distichum). Protoplasma 103:361-375

Drea S, Leader DJ, Arnold BC, Shaw P, Dolan L, Doonan JH (2005) Systematic spatial analysis of gene expression during wheat caryopsis development. Plant Cell 17:2172-2185

Esau K (1977) Anatomy of seed plants, 2nd edn. Wiley, NY

Farley SJ, Patrick JW, Offler CE (2000) Functional transfer cells differentiate in cultured cotyledons of Vicia faba L. seeds. Protoplasma 214:102-117

Gómez E, Royo J, Guo Y, Thompson RD, Hueros G (2002) Establishment of cereal endosperm expression domains: identification and properties of a maize transfer cells-specific transcription factor, ZmMRP-1. Plant Cell 14:599-610

Goverse A, Engler JA, Verhees J, van der Krol S, Helder J, Gheysen G (2000) Cell cycle activation by plant parasitic nematodes. Plant Mol Biol 43:747-761

Gunning BJ, Pate JS, Green LW (1970) Transfer cells in the vascular system of stems: taxonomy, association with nodes, and structure. Protoplasma 71:147-171

Gunning BJ, Pate JS, Minchin FR, Marks I (1974) Quantitative aspects of transfer cells structure in relation to vein loading in leaves and solute transport in legume nodules. Symp Soc Exp Biol 28:87-126

Gutiérrez-Marcos JF, Costa LM, Biderre-Petit C, Khbaya B, O'Sullivan DM, Wormald M, Perez P, Dickinson HG (2004) Maternally expressed gene1 is a novel maize endosperm transfer cell-specific gene with a maternal parent-of-origin pattern of expression. Plant Cell 16:1288-1301

Haritatos E, Medville R, Turgeon R (2000) Minor vein structure and sugar transport in Arabidopsis thaliana. Planta 211:105-111

Hueros G, Varotto S, Salamini F, Thompson RD (1995) Molecular characterization of BETL-1, a gene expressed in the endosperm transfer cells of maize. Plant Cell 7:747-757

Hueros G, Gomez E, Cheikh N, Edwards F, Weldon M, Salamini F, Thompson RD (1999a) Identification of a promoter sequence from the BETL-1 gene cluster able to confer transfer cell-specific expression in transgenic maize. Plant Physiol 121:1143-1152

Hueros G, Royo J, Maitz M, Salamini F, Thomson RD (1999b) Evidence for factors regulating transfer cell-specific expression in maize endosperm. Plant Mol Biol 41:403-414

Ishida Y, Saito H, Ohta S, Hiei Y, Komari T, Kumashiro T (1996) High efficiency transformation of maize (Zea mays L.) mediated by Agrobacterium tumefaciens. Nat Biotechnol 14:745-750

Jefferson RA, Kavanagh TA, Bevan M (1987) GUS fusions: betaglucuronidase as a sensitive and versatile gene fusion marker in higher plants. EMBO J 6:3901-3907

Jones MGK, Northcote DH (1972) Multinucleate transfer cells induced in coleus roots by the root-knot nematode, Meloidogyne arenaria. Protoplasma 75:381-395
Keil M, Sánchez-Serrano JJ, Willmitzer L (1989) Both wound-inducible and tuber-specific expression are mediated by the promoter of a single member of the potato proteinase inhibitor II gene family. EMBO J 8:1323-1330

Kiesselbach TA (1949) The structure and reproduction of corn. Cold Spring Harbor Laboratory Press, Cold Spring Harbor

Komari T, Hiei Y, Saito Y, Murai N, Kumashiro T (1996) Vectors carrying two separate T-DNAs for co-transformation of higher plants mediated by Agrobacterium tumefaciens and segregation of transformants free from selection markers. Plant J 10:165-174

Ligrone R, Gambardella R (1988) The sporophyte-gametophyte junction in bryophytes. Adv Bryol 3:225-274

Muñiz LM, Royo J, Gómez E, Barrero C, Bergareche D, Hueros G (2006) The maize transfer cell-specific type-A response regulator ZmTCRR-1 appears to be involved in intercellular signalling. Plant J 48:17-27

Offler CE, Patrick JW (1993) Pathway of photosynthate transfer in the developing seed of Vicia faba L. A structural assessment of the role of transfer cells in unloading from the seed coat. J Exp Bot 40:711-724

Offler CE, Liet E, Sutton EG (1997) Transfer cell induction in cotyledons of Vicia faba L. Protoplasma 200:51-64

Offler CE, McCurdy DW, Patrick JW, Talbot MJ (2002) Transfer cells: cells specialized for a special purpose. Annu Rev Plant Biol $54: 431-454$

Olsen O-A (2004) Nuclear endosperm development in cereals and Arabidopsis thaliana. Plant Cell 16:S214-S227

Opsahl-Ferstadt H, Le Deunff E, Dumas C, Rogowsky PM (1997) ZmEsr1, a novel endosperm-specific gene expressed in a restricted region around the maize embryo. Plant J 12:235-246

Pate JS, Gunning ES (1972) Transfer cells. Annu Rev Plant Physiol 23:173-196

Pate JS, Gunning ES, Milliken FF (1970) Function of transfer cells in the nodal region of stems, particularly in relation to the nutrition of young seedling. Protoplasma 71:313-334

Pilot G, Stransky H, Bushey DF, Pratelli R, Ludewig U, Wingate VPM, Frommer WB (2004) Overexpression of glutamine dumperl leads to hypersecretion of glutamine from hydathodes of Arabidopsis leaves. Plant Cell 16:1827-1840

Royo J, Gómez E, Hueros G (2007) Transfer cells. In: Olsen O-A (ed) Endosperm- development and molecular biology. Plant Cell Monographs series. Springer-Verlag, Berlin

Sambrook J, Fristsch EF, Maniatis T (1989) Molecular cloning: a laboratory manual, 2nd edn. Cold Spring Harbour Laboratory Press, Cold Spring Harbour

Serna A, Maitz M, O'Connell T, Santandrea G, Thevissen K, Tienens K, Hueros G, Faleri C, Cai G, Lottspeich F, Thompson RD (2001) Maize endosperm secretes a novel antifungal protein into adjacent maternal tissue. Plant J 25:687-698

Sijmons PC (1993) Plant-nematode interactions. Plant Mol Biol 23:917-931

Talbot MJ, Franceschi VR, McCurdy DW, Offler CE (2001) Wall ingrowth architecture in epidermal transfer cells of Vicia faba cotyledons. Protoplasma 215:191-203

Thompson RD, Hueros G, Becker H, Maitz M (2001) Development and functions of seed transfer cells. Plant Sci 160:775-783

Wang N, Fisher DB (1994) The use of fluorescent tracers to characterize de post-phloem transport pathway in maternal tissues of developing wheat grains. Plant Physiol 104:1299-1313

Wang M-B, Li Z-Y, Matthews PR, Upadhyaya NM, Waterhouse PM (1998) Improved vectors for Agrobacterium tumefaciens-mediated transformation of monocot plants. Acta Horticulturae 461:401-405

Weber H, Borijuk L, Heim V, Sauer N, Wobus V (1997) A role for sugar transporter during seed development: molecular characterization of a hexose and sucrose carrier in faba bean seeds. Plant Cell 9:895-908 
Weigel D, Glazebrook J (2002) Arabidopsis: a laboratory manual. Cold Spring Harbour Laboratory Press, Cold Spring Harbour

Weschke W, Panitz R, Gubatz S, Wang Q, Radchuk R, Weber H, Wobus $U$ (2003) The role of invertases and hexose transporters in controlling sugar ratios in maternal and filial tissues of barley caryopses during early development. Plant J 33:395-411

Williams LE, Lemoine R, Saber N (2000) Sugar transporter in higher plants: a diversity of roles and complex regulation. Trends Plant Sci 5:283-290
Wu C, Leubner-Metzger G, Meins FJr, Bradford KJ (2001) Class I $\beta$-1,3-glucanase and chitinase are expressed in the micropylar endosperm of tomato seeds prior to radicle emergence. Plant Physiol 126:1299-1313

Zee SY, O'Brien TP (1971) Vascular transfer cells in the wheat spikelet. Aust J Biol Sci 24:35-49 